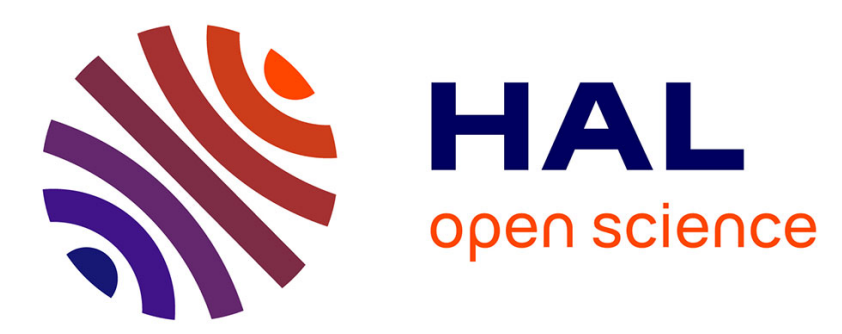

\title{
A "quasi-elastic" affine formulation for the homogenised behaviour of nonlinear viscoelastic polycrystals and composites
}

\author{
R. Brenner, Renaud Masson, O. Castelnau, A. Zaoui
}

\section{- To cite this version:}

R. Brenner, Renaud Masson, O. Castelnau, A. Zaoui. A "quasi-elastic" affine formulation for the homogenised behaviour of nonlinear viscoelastic polycrystals and composites. European Journal of Mechanics - A/Solids, 2002, 21 (6), pp.943-960. 10.1016/S0997-7538(02)01247-0 . hal-03427481

\author{
HAL Id: hal-03427481 \\ https://hal.science/hal-03427481
}

Submitted on 13 Dec 2021

HAL is a multi-disciplinary open access archive for the deposit and dissemination of scientific research documents, whether they are published or not. The documents may come from teaching and research institutions in France or abroad, or from public or private research centers.
L'archive ouverte pluridisciplinaire HAL, est destinée au dépôt et à la diffusion de documents scientifiques de niveau recherche, publiés ou non, émanant des établissements d'enseignement et de recherche français ou étrangers, des laboratoires publics ou privés. 


\title{
A "quasi-elastic" affine formulation for the homogenised behaviour of nonlinear viscoelastic polycrystals and composites
}

\author{
R. Brenner ${ }^{\mathrm{a}, *}$, R. Masson ${ }^{\mathrm{b}}$, O. Castelnau ${ }^{\mathrm{a}}, \mathrm{A}^{\mathrm{A}}$. Zaoui ${ }^{\mathrm{c}}$ \\ a LPMTM-CNRS, Université Paris-Nord, 93430 Villetaneuse, France \\ ${ }^{\mathrm{b}}$ EDF - Research and Development Division, 77818 Moret sur Loing, France \\ ${ }^{\mathrm{c}}$ LMS, CNRS - École polytechnique, 91128 Palaiseau cedex, France
}

Received 11 December 2001; revised and accepted 5 July 2002

\begin{abstract}
The derivation of the overall behaviour of nonlinear viscoelastic (or rate-dependent elastoplastic) heterogeneous materials requires a linearisation of the constitutive equations around uniform per phase stress (or strain) histories. The resulting Linear Comparison Material (LCM) has to be linear thermoviscoelastic to fully retain the viscoelastic nature of phase interactions. Instead of the exact treatment of this LCM (i.e., correspondence principle and inverse Laplace transforms) as proposed by the "classical" affine formulation, an approximate treatment is proposed here. First considering Maxwellian behaviour, comparisons for a single phase as well as for two-phase materials (with "parallel" and disordered morphologies) show that the "direct inversion method" of Laplace transforms, initially proposed by Schapery (1962), has to be adapted to fit correctly exact responses to creep loading while a more general method is proposed for other loading paths. When applied to nonlinear viscoelastic heterogeneous materials, this approximate inversion method gives rise to a new formulation which is consistent with the classical affine one for the steady-state regimes. In the transient regime, it leads to a significantly more efficient numerical resolution, the LCM associated to the step by step procedure being no more thermoviscoelastic but thermoelastic. Various comparisons for nonlinear viscoelastic polycrystals responses to creep as well as relaxation loadings show that this "quasi-elastic" formulation yields results very close to classical affine ones, even for high contrasts.

(c) 2002 Éditions scientifiques et médicales Elsevier SAS. All rights reserved.
\end{abstract}

Keywords: Nonlinear viscoelasticity; Polycrystal; Homogenisation

\section{Introduction}

During the last decades, several attempts have been made to predict the overall response of composites or polycrystals from that of their constituent phases when the latter obeys a nonlinear viscoelastic (or rate-dependent elastoplastic) behaviour. After the first approximate treatments proposed in the seventies by Brown (1970) or Zaoui (1972), several developments for small strains (Weng, 1981) or finite strains (Nemat-Nasser and Obata, 1986; Harren, 1991) were based on an extension of Kröner's conception of the self-consistent approach for elastoplasticity (Kröner, 1961), according to which the viscoplastic strain is considered as a stress-free strain in the sense of Eshelby. It has been proved (Zaoui and Raphanel, 1991) that, such as for elastoplasticity, this approach still deals with elastic interactions between the phases and leads then to too stiff overall responses.

An important specificity with respect to elastoplasticity lies in the fact that the viscoelastic coupling is responsible for a complex "long range memory effect" (Suquet, 1985) which, for instance, shows itself in the non-Maxwellian overall behaviour

\footnotetext{
* Corresponding author.

E-mail address: rb@galilee.univ-paris13.fr (R. Brenner).
} 
of a two-phase material with Maxwellian constituents. Various propositions have been made to deal with this characteristic feature. Generally, they are based either on ad hoc concentration equations (Weng, 1993; Turner et al., 1994; Molinari et al., 1997) or on an internal variables procedure (Paquin et al., 1999). To extend to the nonlinear context the pioneering work of Laws and McLaughlin (1978) proposed for linear viscoelasticity, an alternative approach based on the use of the correspondence principle (Mandel, 1966) and the Laplace transform technique has been proposed by Masson and Zaoui (1999). This theory, which extends to rate-dependent elastoplasticity the tangent linearisation treatment of the local constitutive equations introduced by Molinari et al. (1987) for viscoplasticity, makes use of a homogenisation procedure proposed by Rougier et al. (1994). The resulting so-called "affine" model retains the viscoelastic nature of the interactions between the constituents and the inherent long range memory effect. For linear behaviours, it is consistent with the previous results of Laws and McLaughlin (1978). For nonlinear behaviours, it tends in the steady-state regime to the affine procedure for viscoplasticity (Masson et al., 2000). Nevertheless, although tractable, it has to make use of a complex and computer time-consuming numerical resolution of the inverse Laplace transformation through a collocation method. This method has already been promoted by Schapery (1962) (and also frequently applied in linear viscoelasticity, e.g., Turner and Tomé (1993)) who proposed at the same time a powerful approximation referred to as the "direct method".

The main practical aim of this paper is a systematic application of this last method to the affine modelling of the overall behaviour of nonlinear viscoelastic polycrystals and composites. We first (Section 2) recall the essential points of the affine procedure for this class of materials. In Section 3, we analyse different ways to make use of the "direct method" for homogeneous and heterogeneous linear viscoelasticity and different applied strain and stress paths. We then propose (Section 4) a "quasi-elastic" approximation of the affine formulation for nonlinear heterogeneous viscoelasticity and we show in Section 5 that it provides a very satisfying tool for the prediction of the creep and relaxation behaviour of polycrystals. Further progress are briefly discussed in the conclusion.

\section{The affine procedure for nonlinear viscoelastic materials}

\subsection{The linearisation procedure}

Homogenisation of the nonlinear behaviour of heterogeneous materials generally resorts to the linearisation of the local constitutive equations before applying some scale change scheme. This is also the case for nonlinear viscoelasticity. For such a behaviour, variational procedure (see Ponte Castañeda, 1991, 1996; Suquet, 1993) aiming at the construction of bounds or estimates cannot be invoked since the constitutive equations do not derive from a single potential, neither at the local nor at the global scale. In this context, for which only estimates can be investigated, an alternative approach allowing to describe the coupling between conservative (elastic) and dissipative (viscous) regimes has to be adopted. The affine procedure (Masson and Zaoui, 1999), developed in that goal, has been chosen for the present study.

Let us consider a multiphase nonlinear viscoelastic medium, occupying a volume $\Omega$, whose phases obey constitutive equations which read, for infinitesimal strains, at any time $t$

$$
\dot{\boldsymbol{\varepsilon}}(\mathbf{x}, t)=\mathbf{s}(\mathbf{x}): \dot{\boldsymbol{\sigma}}(\mathbf{x}, t)+\mathbf{g}(\mathbf{x}, \boldsymbol{\sigma}(\mathbf{x}, t)), \quad \forall \mathbf{x} \in \Omega,
$$

with $\dot{\boldsymbol{\varepsilon}}$ and $\dot{\boldsymbol{\sigma}}$ the strain and stress rates, $\mathbf{s}$ the elastic compliance and $\mathbf{g}$ the nonlinear viscous (or viscoplastic) strain rate deriving from the potential $u$. For the sake of simplicity (but this is by no means a limitation), a sole dependence of $\mathbf{g}$ on the local stress state $\sigma(\mathbf{x}, t)$ is considered:

$$
\mathbf{g}(\mathbf{x}, \boldsymbol{\sigma}(\mathbf{x}, t))=\frac{\partial u}{\partial \boldsymbol{\sigma}}(\mathbf{x}, \boldsymbol{\sigma}(\mathbf{x}, t)) .
$$

The material is supposed to be subjected to a known macroscopic mechanical loading for $t \in[0 ;+\infty[$. For brevity, the position vector $\mathbf{x}$ is omitted afterwards.

The main difficulty of the linearisation step is related to the differential nature of relation (1). Indeed, unlike elastoplasticity or viscoplasticity, it does not allow us to define directly instantaneous moduli or compliances since the strain rate at each time $t$ depends on the whole stress history from $t=0$. Consequently, associated to the linearisation of the strain rate $\dot{\varepsilon}$ at a prescribed time $t=\zeta$, it is necessary to know its expression from $t=0$ to $t=\zeta$. This linearisation, proposed by Rougier et al. (1994), reads

$$
\dot{\boldsymbol{\varepsilon}}(t)=\mathbf{s}: \dot{\boldsymbol{\sigma}}(t)+\mathbf{m}(\zeta): \boldsymbol{\sigma}(t)+\dot{\boldsymbol{\varepsilon}}^{0}(t, \zeta), \quad \mathbf{m}(\zeta)=\frac{\partial \mathbf{g}}{\partial \boldsymbol{\sigma}}(\zeta)
$$

with

$$
\begin{cases}\dot{\boldsymbol{\varepsilon}}^{0}(t, \zeta)=\mathbf{g}(\boldsymbol{\sigma}(t))-\mathbf{m}(\zeta): \boldsymbol{\sigma}(t) & \forall t \leqslant \zeta \\ \dot{\boldsymbol{\varepsilon}}^{0}(t, \zeta)=\mathbf{g}(\boldsymbol{\sigma}(\zeta))-\mathbf{m}(\zeta): \boldsymbol{\sigma}(\zeta) & \forall t \geqslant \zeta\end{cases}
$$


which is an approximation of a more general procedure proposed by Pouya an Zaoui (1999). It can be noted that the stress-free strain rate $\dot{\boldsymbol{\varepsilon}}^{0}(t, \zeta)$ does not depend on the mechanical loading after $t=\zeta$. With use of the notion of the "linear composite comparison medium" (Ponte Castañeda, 1991), we can say that according to (3) and (4), this procedure refers to a linear thermoviscoelastic heterogeneous medium. Relation (3) can be put in the more convenient form of a Stieljes convolution product:

$$
\left\{\begin{array}{l}
\boldsymbol{\varepsilon}(t)=\int_{0}^{t} \mathbf{q}_{\zeta}(t-u): \mathrm{d} \boldsymbol{\sigma}(u)+\boldsymbol{\varepsilon}^{0}(t, \zeta) \\
\mathbf{q}_{\zeta}(t)=\mathbf{s}+\mathbf{m}(\zeta) t \\
\boldsymbol{\varepsilon}^{0}(t, \zeta)=\int_{0}^{t} \dot{\boldsymbol{\varepsilon}}^{0}(\theta, \zeta) \mathrm{d} \theta
\end{array}\right.
$$

The stress-free strain $\varepsilon^{0}$ is a function of the whole stress history thus accounting for the viscoelastic coupling phenomena within the material. At last, we recall that the choice of a sole dependence of the potential $u$ on the stress $\sigma$ in (2) is not a restriction of the procedure. The introduction of internal variables which are necessary to take into account microstructural evolutions (hardening, texture evolution, etc.) within this scheme is fully manageable. For a complete derivation, the reader is referred to Masson and Zaoui (1999).

\subsection{The mean field approximation}

Hereafter, the material is assumed to be composed of $N$ phases $(r)$, occupying volumes $\Omega_{r}$, with a given microstructure described by the characteristic functions $\chi_{r}(\mathbf{x})$ equal to 1 if $\mathbf{x} \in \Omega_{r}$ and 0 otherwise. In the following, the microstructure is only considered in a statistical way through the first-order (e.g., volume fractions) and second-order (e.g., covariances) correlation functions.

To derive the homogenised properties, the linearisation procedure defined above would have to be applied at each point $\mathbf{x}$ so as to determine the local stress fields $\boldsymbol{\sigma}(\mathbf{x})$. Obviously, such a problem cannot be addressed without some simplification, since it would require a complete knowledge of the characteristic functions in order to obtain the stress concentration tensors at each point $\mathbf{x}$. Consequently, the usual mean field approximation has to be used. It consists, using the statistical description of the microstructure, in the determination of the phase stress and strain averages, $\langle\boldsymbol{\sigma}\rangle_{r}$ and $\langle\boldsymbol{\varepsilon}\rangle_{r}$, e.g., for Hashin-Shtrikmantype estimates, through the use of Eshelby's solution (Eshelby, 1957) of the inclusion problem. For this step, the affine method classically defines the linearised behaviour (compliance and stress-free strain) with respect to the average fields over each phase. This choice, originally made by Hill (1965) for elastoplasticity, implies that the stress and strain phase averages are related by the constitutive equations (1). At any linearisation time $\zeta$, the affine behaviour (5) reduces to

$$
\left\{\begin{array}{l}
\boldsymbol{\varepsilon}_{r}(t)=\left[\mathbf{q}_{\zeta} * \boldsymbol{\sigma}_{r}\right](t)+\boldsymbol{\varepsilon}_{r}^{0}(t, \zeta) \\
\mathbf{q}_{\zeta}(t)=\mathbf{s}+\mathbf{m}(\zeta) t, \quad \mathbf{m}(\zeta)=\frac{\partial \mathbf{g}}{\partial \boldsymbol{\sigma}}\left(\boldsymbol{\sigma}_{r}(\zeta)\right)
\end{array}\right.
$$

where $*$ denotes the Stieltjes convolution product and introducing the notation ${ }_{r}=\langle\cdot\rangle_{r}$. It has to be mentioned that refined definitions of the linearised behaviour taking into account, for instance, the intraphase second moment of the fields as proposed by Brenner et al. (2001) could be used in (6). This possibility will be explored in a future work.

To obtain the homogenised properties at time $t=\zeta$, the correspondence principle can be used. It helps to transform the linear thermo-viscoelastic behaviour into a symbolic thermoelastic one for which the classical homogenisation techniques apply. For that goal, the use of the Laplace-Carson transform, namely

$$
\hat{f}(p)=p \int_{0}^{+\infty} f(t) \mathrm{e}^{-p t} \mathrm{~d} t
$$

where $p$ is the complex variable, is especially convenient. Applied to (6), it leads to

$$
\hat{\boldsymbol{\varepsilon}}_{r}(p)=\hat{\mathbf{q}}_{\zeta}(p): \hat{\boldsymbol{\sigma}}_{r}(p)+\hat{\boldsymbol{\varepsilon}}_{r}^{0}(p), \quad \hat{\mathbf{q}}_{\zeta}(p)=\mathbf{s}+\frac{1}{p} \mathbf{m}(\zeta) .
$$

The symbolic overall properties are then classically given by

$$
\left\{\begin{array}{l}
\widehat{\mathbf{Q}}_{\zeta}(p)=\left\langle\hat{\mathbf{q}}_{\zeta}(p): \widehat{\mathbf{B}}_{r}(p, \zeta)\right\rangle \\
\widehat{\mathbf{E}}^{0}(p, \zeta)=\left\langle{ }^{t} \widehat{\mathbf{B}}_{r}(p, \zeta): \hat{\boldsymbol{\varepsilon}}_{r}^{0}(p, \zeta)\right\rangle
\end{array}\right.
$$


where $\langle\cdot\rangle$ denotes the volume average over $\Omega, \widehat{\mathbf{B}}_{r}$ is the symbolic stress concentration tensor over phase $(r), \widehat{\mathbf{Q}}_{\zeta}$ is the symbolic effective compliance and $\widehat{\mathbf{E}}^{0}$ is the symbolic effective stress-free strain. The $\widehat{\mathbf{B}}_{r}$ tensors depend on the homogenisation scheme adopted, according to the structural morphology of the considered material.

Once the overall symbolic properties are obtained, it is necessary to proceed to their Laplace-Carson inversion in order to get the homogenised behaviour in the direct time space. Except for very particular cases (see Section 3), analytical expressions of the different symbolic quantities $\left(\widehat{\mathbf{Q}}(p), \widehat{\mathbf{B}}_{r}(p)\right.$, etc.) are not available. As a consequence, their inverse transforms must be performed numerically. To do that, Masson and Zaoui chose the collocation method (Schapery, 1962) which approximates the transient part of any viscoelastic function by a finite Dirichlet series. From the values of the symbolic function to invert at $M$ collocation points $p$ in the Laplace-Carson space, the inverse transform is computed using a least square minimisation with respect to the $M$ coefficients of the series. The main advantage of this method lies in the control of the accuracy of the inversion which can be improved with an increasing number of collocation points. For the considered case, it requires in practice about 20 relaxation times equispaced over six decades on a logarithmic time scale (Turner and Tomé, 1993). The counterpart of the method is that it requires, at each time $\zeta$, as many resolution operations of the homogenisation equations (9) as collocation points. Though tractable, this implies CPU time-consuming computations. These observations motivate the study of an alternative approximate treatment in view of faster computations. This treatment is now first defined for linear viscoelasticity (Section 3) and then applied to the affine modelling of nonlinear heterogeneous viscoelasticity (Section 4).

\section{An approximate treatment for linear viscoelasticity}

\subsection{The direct inversion method}

The "direct inversion method" is an approximate method of Laplace-Carson (LC) transform inversion initially proposed by Schapery (1962). It is based on a mathematical property of the LC transform (7) which, using the change of variables $w=\log _{10}(p t)$, reads alternatively

$$
\left\{\begin{array}{l}
\hat{f}(p)=\int_{-\infty}^{+\infty} f\left(\frac{10^{w}}{p}\right) g(w) \mathrm{d} w, \\
g(w)=10^{w} \mathrm{e}^{-10^{w}} \log _{\mathrm{e}} 10 .
\end{array}\right.
$$

The key-point of the direct inversion method lies in the form of the function $g(w)$ which is quasi-null everywhere except for $-2 \leqslant w \leqslant 1$ (Fig. 1) and satisfies

$$
\int_{-\infty}^{+\infty} g(w) \mathrm{d} w=1 .
$$

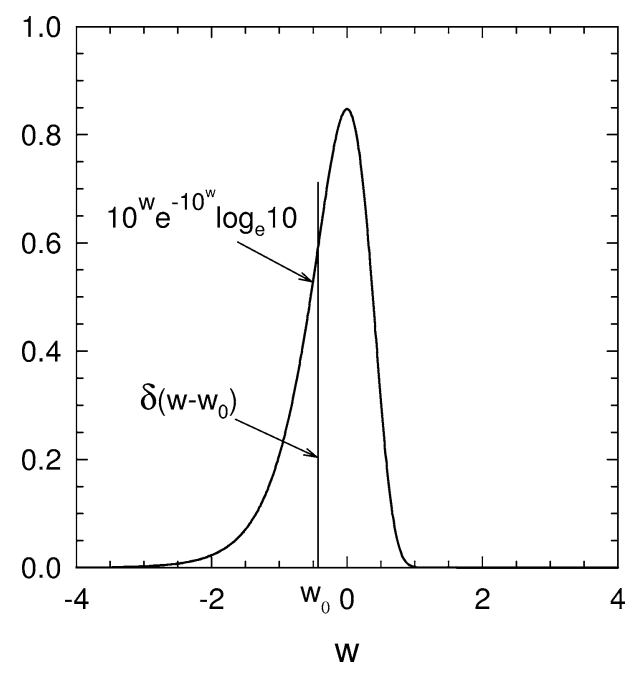

Fig. 1. Shape of the function $g(w)$ defined by Eq. (10). 
It can thus be advantageously replaced, as a first approximation, by a Dirac function $\delta\left(w-w_{0}\right)$. This leads to the following relation

$$
\hat{f}(p) \simeq f(t)_{t=10^{w_{0}} / p} \text { or, inversely, } \quad f(t) \simeq \hat{f}(p)_{p=10^{w_{0}} / t} .
$$

Thus, knowing the value of $\hat{f}$ for a single value of $p=10^{w_{0}} / t$ is sufficient for an approximate estimation of $f(t)$. To determine the optimal value of $w_{0}$, it is necessary to consider the shape of $f$. For functions presenting a quasi-linear variation versus $\log (t)$, Schapery obtained the value $w_{0}=-C / \log _{\mathrm{e}} 10$ with $C$ the Euler constant. The method becomes exact if the considered function is rigorously proportional to $\log (t)$. Schapery further proposed to use an approximate value for $C$ leading to $10^{w_{0}}=0.5$. Therefore, the initially proposed direct method provides a reasonable approximation if the function $f(t)$ presents a quasi-linear behaviour over a range of 3 decades on a logarithmic time scale, due to the form of $g(w)$. For polycrystals, according to Turner et al. (1994), the stress and strain responses as well as creep compliances are smooth functions of time and can therefore be transformed in accordance with the so-defined direct method. The authors further concluded that this approximate treatment is limited to responses that do not exhibit rapid variations in time, such as creep deformation tests.

In this section, restriction is made to linear viscoelasticity in order to reconsider, on simpler situations, the most appropriate value for $w_{0}$ for the coming applications which are mainly focussed on the creep response of nonlinear viscoelastic heterogeneous materials. The way other deformation paths (i.e., more complex than creep) may be treated will also be indicated.

\subsection{Creep response}

For sake of simplicity, we consider in the following an isotropic material whose constituents obey an isotropic incompressible Maxwellian behaviour defined by

$$
\dot{\boldsymbol{\varepsilon}}(t)=\mathrm{m}_{i}^{\mathrm{e}} \dot{\boldsymbol{\sigma}}^{\prime}(t)+\mathrm{m}_{i}^{\mathrm{v}} \boldsymbol{\sigma}^{\prime}(t)
$$

The scalar constants $\mathrm{m}_{i}^{\mathrm{e}}$ and $\mathrm{m}_{i}^{\mathrm{v}}$ are, respectively, the elastic and viscous shear compliances of phase $i$, $\dot{\boldsymbol{\varepsilon}}$ is isochoric and $\sigma^{\prime}$ is the stress deviator. Thus, the relaxation spectrum reduces to a single relaxation time $\tau_{i}=\mathrm{m}_{i}^{\mathrm{e}} / \mathrm{m}_{i}^{\mathrm{v}}$. The overall behaviour of this heterogeneous material is known to be no more Maxwellian, due to the long range memory effect. Relation (13) can be given in the following integral form

$$
\boldsymbol{\varepsilon}(t)=\left[\mathrm{m}_{i}^{\mathrm{ve}} * \boldsymbol{\sigma}^{\prime}\right](t)
$$

with $\mathrm{m}_{i}^{\mathrm{ve}}(t)=\mathrm{m}_{i}^{\mathrm{e}}+t \mathrm{~m}_{i}^{\mathrm{V}}$. Using the LC transform technique, relation (14) can be transformed into

$$
\hat{\boldsymbol{\varepsilon}}(p)=\widehat{\mathrm{m}}_{i}^{\mathrm{ve}}(p) \hat{\boldsymbol{\sigma}}^{\prime}(p)
$$

with $\widehat{\mathrm{m}}_{i}^{\mathrm{ve}}(p)=\mathrm{m}_{i}^{\mathrm{e}}+(1 / p) \mathrm{m}_{i}^{\mathrm{v}}$ or equivalently:

$$
\hat{\boldsymbol{\sigma}}^{\prime}(p)=2 \hat{\mu}_{i}(p) \hat{\boldsymbol{\varepsilon}}(p), \quad \hat{\mu}_{i}(p)=\frac{p}{2 \mathrm{~m}_{i}^{\mathrm{e}}\left(p+1 / \tau_{i}\right)} .
$$

The viscoelastic behaviour (14) is transformed into a symbolic elastic behaviour (15) in the LC space in which classical linear homogenisation schemes apply. The response is obtained in the LC space and it has then to be inverse transformed. For this last step, we analyse the consequences of the approximate method evoked above.

Let us consider a creep loading under homogeneous boundary conditions at $t=0$ of the form $\Sigma^{\prime}=\mathbf{S}_{0} H(t)$, with $H(t)$ the Heaviside function and $\Sigma^{\prime}$ the overall stress deviator. We start with the most simple case of a homogeneous material, i.e., consisting in a single phase, so that $\sigma^{\prime}(t)=\Sigma^{\prime}$. The strain response reads, for $t>0$

$$
\boldsymbol{\varepsilon}(t)=\left(\mathrm{m}^{\mathrm{e}}+t \mathrm{~m}^{\mathrm{v}}\right) \mathbf{S}_{0} \stackrel{\mathcal{L C}}{\longrightarrow} \hat{\boldsymbol{\varepsilon}}(p)=\left(\mathrm{m}^{\mathrm{e}}+\frac{1}{p} \mathrm{~m}^{\mathrm{v}}\right) \mathbf{S}_{0},
$$

where $\mathcal{L C}$ denotes the LC transform. So, $\boldsymbol{\varepsilon}$ is linear with respect to $t$ and not to $\log (t)$ as considered by Schapery and Turner et al. It follows from (17) that the direct method leads in that case to the exact response only for $10^{w_{0}}=1$ (i.e., $p=1 / t$ in $(12)$ ) instead of $10^{w_{0}}=0.5$ as proposed initially, which would give a twice too large creep rate. It must also be remarked that the choice $10^{w_{0}}=1$ is the one made implicitly by Turner at al. (1994) whereas they were supposed to use $10^{w_{0}}=0.5$ according to their reference to Schapery (1962).

The effect of the direct inversion method applied on a heterogeneous material may be more difficult to analyse since, in that case, not only the shape of the local strain response has to be considered, but also the evolution with time of all other quantities such as local stress and localisation tensors. For this reason, we still consider the relatively simple case of a two-phase material. The local strain reads, according to the direct method: 


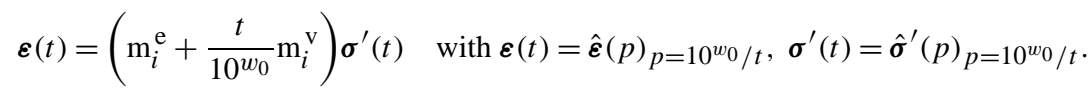

Important is to recognise that the application of the direct method leads to a linear (time-dependent) problem that can be treated directly in the time space with classical homogenisation schemes, and it is therefore very efficient numerically. Note however that some long range memory effect is still considered in this so-called "quasi-elastic" approximation, as shown by the fact that the overall behaviour is not Maxwellian. It can also be remarked that it is equivalent to apply the direct method to the quantities homogenised in the LC space with local behaviour given by (15) or to carry out homogenisation directly in the time space with local behaviour defined by (18).

The error introduced by the direct inversion method can be estimated by comparing the time derivative of (18)

$$
\dot{\boldsymbol{\varepsilon}}(t)=\left(\mathrm{m}_{i}^{\mathrm{e}}+\frac{t}{10^{w_{0}}} \mathrm{~m}_{i}^{\mathrm{v}}\right) \dot{\boldsymbol{\sigma}}^{\prime}(t)+\frac{1}{10^{w_{0}}} \mathrm{~m}_{i}^{\mathrm{v}} \boldsymbol{\sigma}^{\prime}(t)
$$

with Eq. (13). The direct method introduces an extra time-dependent elastic compliance $t \mathrm{~m}_{i}^{\mathrm{V}} / 10^{w_{0}}$ and therefore the Maxwellian character of the local behaviour is lost. Since the effect of this extra term vanishes in the steady-state creep regime $\left(\dot{\boldsymbol{\sigma}}^{\prime}=0\right)$, the steady-state strain rate predicted by the direct method is the exact one for $10^{w_{0}}=1$. In the transient regime, the effect of this term can be investigated by calculating the error $\left(F-F^{\mathrm{DM}}\right) / F$, where $F(t)$ refers to the creep function (defined as $\left.\mathbf{E}(t)=F(t) \boldsymbol{\Sigma}_{0} H(t)\right)$ obtained by the rigourous LC inversion and $F^{\mathrm{DM}}$ the same quantity when applying the direct method.

This error can be expressed easily if one considers the particular microstructure (say, a "parallel" microstructure) which would correspond to a Voigt-type estimation $\boldsymbol{\varepsilon}=\mathbf{E}$, with $\mathbf{E}$ the overall strain. In that case, the exact ${ }^{1}$ and the approximate overall creep functions read respectively:

$$
\left\{\begin{array}{l}
F(t)=\frac{\mathrm{m}_{1}^{\mathrm{e}} \mathrm{m}_{2}^{\mathrm{e}}}{c_{2} \mathrm{~m}_{1}^{\mathrm{e}}+c_{1} \mathrm{~m}_{2}^{\mathrm{e}}}\left\{1+\frac{t}{\bar{\tau}}+\frac{\left(\tau_{2}-\bar{\tau}\right)\left(\bar{\tau}-\tau_{1}\right)}{\bar{\tau}^{2}}\left[1-\mathrm{e}^{-t / \tilde{\tau}}\right]\right\}, \\
F^{\mathrm{DM}}(t)=\frac{\mathrm{m}_{1}^{\mathrm{e}} \mathrm{m}_{2}^{\mathrm{e}}}{c_{2} \mathrm{~m}_{1}^{\mathrm{e}}+c_{1} \mathrm{~m}_{2}^{\mathrm{e}}}\left\{1+10^{-w_{0}} \frac{t}{\bar{\tau}}+\frac{\left(\tau_{2}-\bar{\tau}\right)\left(\bar{\tau}-\tau_{1}\right)}{\bar{\tau}^{2}}\left[1-\frac{1}{1+10^{-w_{0} t / \tilde{\tau}}}\right]\right\}
\end{array}\right.
$$

with $\bar{\tau}=\left(c_{1} \mathrm{~m}_{2}^{\mathrm{e}} \tau_{1}+c_{2} \mathrm{~m}_{1}^{\mathrm{e}} \tau_{2}\right) /\left(c_{2} \mathrm{~m}_{1}^{\mathrm{e}}+c_{1} \mathrm{~m}_{2}^{\mathrm{e}}\right)$ and $\tilde{\tau}=\tau_{1} \tau_{2} / \bar{\tau}$. Corresponding results are plotted in Fig. 2 for $10^{w_{0}}=0.5$ and $10^{w_{0}}=1$, different ratios $\tau_{2} / \tau_{1}$ of the relaxation times of the two phases, identical volume fractions $c_{i}$ of both phases, and $k=\mathrm{m}_{1}^{\mathrm{e}} / \mathrm{m}_{2}^{\mathrm{e}}=1$. As discussed above for the homogeneous material, taking $10^{w_{0}}=1$ greatly improves the approximation as compared to the original proposition of Schapery, and this for both transient and steady-state regimes. As shown in Fig. 2, the error increases, for $10^{w_{0}}=1$, with increasing values of $\tau_{2} / \tau_{1}$. Indeed, it is worth noting that when the two phases have identical relaxation times, the ratio $\widehat{\mathrm{m}}_{1}^{\mathrm{ve}} / \widehat{\mathrm{m}}_{2}^{\mathrm{ve}}$ of the symbolic compliances does not depend on $p$ and equals $\mathrm{m}_{1}^{\mathrm{e}} / \mathrm{m}_{2}^{\mathrm{e}}$. It can then easily

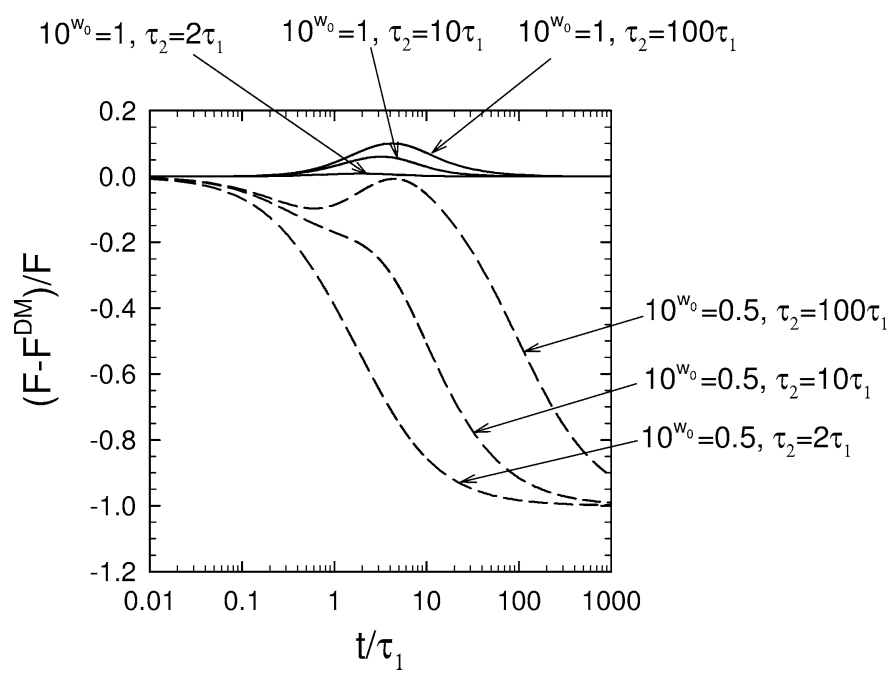

Fig. 2. Normalised difference between the exact creep function and the one predicted by the quasi-elastic approximation for $10^{w_{0}}=0.5,10^{w_{0}}=1$, and several ratios $\tau_{2} / \tau_{1}$, in the case of a two-phase material with "parallel" Maxwellian phases $\left(c_{2}=0.5, k=1\right)$.

\footnotetext{
${ }^{1}$ In the sequel, the term "exact" supposes that the microstructure realises the adopted linear scheme.
} 
be proved that in that case, the local stresses are constant during the whole creep test, and consequently the direct method gives the exact LC inversion as for a single phase material. The accuracy of the direct method is therefore especially dependent on the heterogeneity of the relaxation times of the different phases.

For the coming applications to polycrystals, a more realistic microstructure has to be considered. As an illustration, the overall behaviour and local mechanical fields are estimated through the classical self-consistent scheme, for which the quasielastic approximation can be compared to the exact solution. According to Rougier et al. (1993) or Beurthey and Zaoui (2000), the overall relaxation shear modulus $\tilde{\mu}(t)$ can be derived from its Laplace-Carson transform $\hat{\tilde{\mu}}(p)$ which obeys the second-order equation

$$
\widehat{X}^{2}+\left[\frac{(2-5 c) k\left(p+1 / \tau_{1}\right)}{3\left(p+1 / \tau_{2}\right)}+\frac{5 c-3}{3}\right] \widehat{X}-\frac{2 k\left(p+1 / \tau_{1}\right)}{3\left(p+1 / \tau_{2}\right)}=0
$$

with $\widehat{X}=\hat{\tilde{\mu}} / \hat{\mu}_{1}$ and $c=c_{2}$. The solution $\hat{\tilde{\mu}}(p)$ reads

$$
\left\{\begin{array}{l}
12 \mathrm{~m}_{1}^{\mathrm{e}} \hat{\tilde{\mu}}(p)=\frac{(5 c-3) p}{p+1 / \tau_{1}}+\frac{(5 c-2) p}{p+1 / \tau_{2}}+K \frac{p[P(p)]^{1 / 2}}{\left(p+1 / \tau_{1}\right)\left(p+1 / \tau_{2}\right)}, \\
K=\left[(2-5 c)^{2} k^{2}+2 k(6-5 c)(5 c+1)+(5 c-3)^{2}\right]^{1 / 2}, \\
P(p)=\left(p+1 / \theta_{1}\right)\left(p+1 / \theta_{2}\right),
\end{array}\right.
$$

where $-1 / \theta_{1}$ and $-1 / \theta_{2}$ are the real negative solutions of the second-order equation

$$
\begin{aligned}
& K^{2} p^{2}+2\left[(2-5 c)^{2} k^{2} / \tau_{1}+k(6-5 c)(5 c+1)\left(1 / \tau_{1}+1 / \tau_{2}\right)+(5 c-3)^{2} / \tau_{2}\right] p \\
& \quad+(2-5 c)^{2}\left(k / \tau_{1}\right)^{2}+2 k(6-5 c)(5 c+1) / \tau_{1} \tau_{2}+(5 c-3)^{2} / \tau_{2}^{2}=0 .
\end{aligned}
$$

From (22) and (23), the overall response to any stress $\boldsymbol{\Sigma}^{\prime}(t)$ or strain $\mathbf{E}(t)$ history can be derived, after inversion of the LaplaceCarson transformation, through the relation $\widehat{\boldsymbol{\Sigma}}^{\prime}(p)=2 \hat{\tilde{\mu}}(p) \widehat{\mathbf{E}}(p)$ whereas the per phase average variables $\hat{\boldsymbol{\varepsilon}}_{i}(p)$ and $\hat{\boldsymbol{\sigma}}_{i}(p)$ are obtained from the macroscopic quantities through the average strain $\hat{\mathbf{A}}_{i}(p)$ or stress $\widehat{\mathbf{B}}_{i}(p)$ concentration tensors, which reduce here, due to isotropy, to the scalar functions $\hat{A}_{i}(p)$ and $\widehat{B}_{i}(p)$, e.g.,

$$
\hat{\boldsymbol{\varepsilon}}_{i}(p)=\hat{A}_{i}(p) \widehat{\mathbf{E}}(p), \quad \hat{\boldsymbol{\sigma}}_{i}(p)=2 \hat{\mu}_{i}(p) \hat{\boldsymbol{\varepsilon}}_{i}(p), \quad \hat{A}_{i}=\frac{5 \hat{\tilde{\mu}}}{3 \hat{\tilde{\mu}}+2 \hat{\mu}_{i}} .
$$

The exact inverse transforms of the symbolic creep functions $\widehat{F}(p)$ and $\hat{f}_{i}(p)$, defined as

$$
\begin{cases}\boldsymbol{\Sigma}^{\prime}(t)=\mathbf{S}_{0} H(t), & \widehat{\mathbf{E}}(p)=\widehat{F}(p) \mathbf{S}_{0}, \quad \hat{\boldsymbol{\varepsilon}}_{i}(p)=\hat{f}_{i}(p) \mathbf{S}_{0}, \\ \widehat{F}(p)=\frac{1}{2 \hat{\tilde{\mu}}(p)}, & \hat{f}_{i}(p)=\frac{\hat{A}_{i}(p)}{2 \hat{\tilde{\mu}}(p)}=\frac{5}{2\left[3 \hat{\tilde{\mu}}(p)+2 \hat{\mu}_{i}(p)\right]}\end{cases}
$$

are given in Appendix A. An illustrative example of the overall and average phase responses for creep is given in Fig. 3 for $\tau_{2}=10 \tau_{1}$, by comparing the exact solutions to their approximations according to the direct inversion method. It is clear that the initial choice of Schapery $\left(10^{w_{0}}=0.5\right)$ does not give satisfactory results, a much better one being obtained when taking $10^{w_{0}}=1$. As shown in Fig. 3(b), the direct method is not only able to predict the overall behaviour with a very good accuracy, but also phase average responses are very well estimated. For the presented case, the strain difference between exact and approximate solutions never exceeds 5\%, neither in the transient nor in the steady-state regime. The origin of this very good agreement lies in the almost constant local stresses for $t>0$.

More generally, according to the known property of the LC transform for the initial and final values, i.e.,

$$
\left\{\begin{array}{l}
\lim _{|p| \rightarrow+\infty} \hat{f}(p)=\lim _{t \rightarrow 0_{+}} f(t), \\
\lim _{p \rightarrow 0} \hat{f}(p)=\lim _{t \rightarrow+\infty} f(t)
\end{array}\right.
$$

the direct method yields exact limits for $f(t)$ when $t \rightarrow 0_{+}$and $t \rightarrow+\infty$ whatever the choice of $\omega_{0}$. But for sufficiently smooth functions $f(t)$, the same property stands near $t=0$ for the time derivative $\dot{f}(t)$ only for $10^{w_{0}}=1$, since the asymptotic behaviour of such functions obeys the relation:

$$
\left\{\begin{array}{l}
\text { if } f(t)=f\left(0_{+}\right)+\dot{f}\left(0_{+}\right) t+\mathcal{O}\left(t^{2}\right), t>0, \\
\text { then } \hat{f}(p)=f\left(0_{+}\right)+\frac{\dot{f}\left(0_{+}\right)}{p}+\mathcal{O}\left(\frac{1}{p^{2}}\right), \operatorname{Re}(p)>0,
\end{array}\right.
$$


(a)

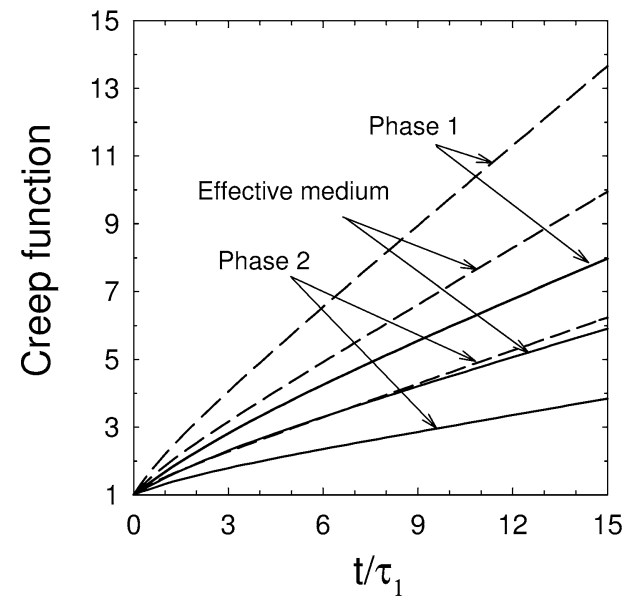

(b)

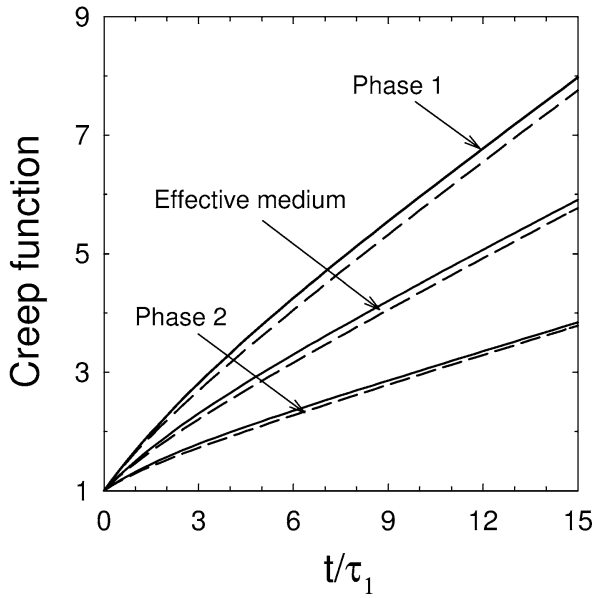

Fig. 3. Local $(f)$ and overall $(F)$ creep functions predicted by the quasi-elastic approximation (dashed lines) as compared to the exact results (continuous lines) for a two-phase material according to the self-consistent scheme, for $\tau_{2} / \tau_{1}=10, c_{2}=0.5, k=1$. (a) $10^{w_{0}}=0.5$ and (b) $10^{w_{0}}=1$.

where $\mathcal{O}(x)$ means "of the order of $x$ ". A similar conclusion stands for $f(t) / t$ for large values of $t$. Nevertheless, as commented below, the practical importance of these properties depends on the values of the involved relaxation times with respect to the observation time interval.

\subsection{Relaxation}

Let us now consider the response of the same material when subjected to a relaxation strain path at $t=0$, i.e., to an overall applied strain $\mathbf{E}(t)=\mathbf{E}_{0} H(t)$.

If the material is homogeneous, the stress response reads, for $t>0$

$$
\boldsymbol{\sigma}^{\prime}(t)=\frac{\mathbf{E}_{0}}{\mathrm{~m}^{\mathrm{e}}} \mathrm{e}^{-t / \tau} \stackrel{\mathcal{L C}}{\longrightarrow} \hat{\boldsymbol{\sigma}}^{\prime}(p)=\frac{\mathbf{E}_{0}}{\mathrm{~m}^{\mathrm{e}}} \frac{\tau p}{1+\tau p} .
$$

It is clear that, in that case, the direct method as defined above does not lead to the exact result whatever the choice of $w_{0}$. A different value for $w_{0}$ may be proposed to minimise the gap between the exact and approximate stress responses, but this value must depend on the relaxation time $\tau$ of the material. Furthermore, the direct method provides at large $t$ a much slower stress decrease as compared to the exact solution, whatever $w_{0}$. Note however that, according to $(27)$, only the value $10^{w_{0}}=1$ provides the exact stress rate at $t=0$.

As for creep, the error introduced by the direct method can be calculated easily for a two-phase material with a "parallel" microstructure. It reads now $\left(R-R^{\mathrm{DM}}\right) / R(0)$, with $R$ the relaxation function defined by $\boldsymbol{\Sigma}^{\prime}(t)=R(t) \mathbf{E}_{0} H(t)$. The exact and approximate overall relaxation functions are given by

$$
\left\{\begin{array}{l}
R(t)=\frac{c_{1}}{\mathrm{~m}_{1}^{\mathrm{e}}} \mathrm{e}^{-t / \tau_{1}}+\frac{c_{2}}{\mathrm{~m}_{2}^{\mathrm{e}}} \mathrm{e}^{-t / \tau_{2}}, \\
R^{\mathrm{DM}}(t)=\frac{c_{1}}{\mathrm{~m}_{1}^{\mathrm{e}}} \frac{1}{1+10^{-w_{0}} t / \tau_{1}}+\frac{c_{2}}{\mathrm{~m}_{2}^{\mathrm{e}}} \frac{1}{1+10^{-w_{0} t / \tau_{2}}} .
\end{array}\right.
$$

Results for $\tau_{2}=2 \tau_{1}$ and $\tau_{2}=10 \tau_{1}$ are shown in Fig. 4. As for the single phase material, the agreement is not satisfactory for both $10^{w_{0}}=0.5$ and $10^{w_{0}}=1$, especially at large reduced time $t / \tau_{1}$ where the stress decrease is really too slow. According to Fig. 5, the same conclusion can be drawn for a disordered microstructure corresponding to the self-consistent scheme for which the exact inverse transforms of local and overall symbolic relaxation functions $\hat{r}_{i}(p)$ and $\widehat{R}(p)$

$$
\left\{\begin{array}{l}
\boldsymbol{E}(t)=\boldsymbol{E}_{0} H(t), \quad \widehat{\boldsymbol{\Sigma}}^{\prime}(p)=\widehat{R}(p) \boldsymbol{E}_{0}, \quad \hat{\boldsymbol{\sigma}}_{i}^{\prime}(p)=\hat{r}_{i}(p) \boldsymbol{E}_{0}, \\
\widehat{R}(p)=2 \hat{\tilde{\mu}}(p), \quad \hat{r}_{i}(p)=2 \hat{\mu}_{i}(p) \hat{A}_{i}(p)=\frac{10 \hat{\tilde{\mu}}(p) \hat{\mu}_{i}(p)}{3 \hat{\tilde{\mu}}(p)+2 \hat{\mu}_{i}(p)}
\end{array}\right.
$$



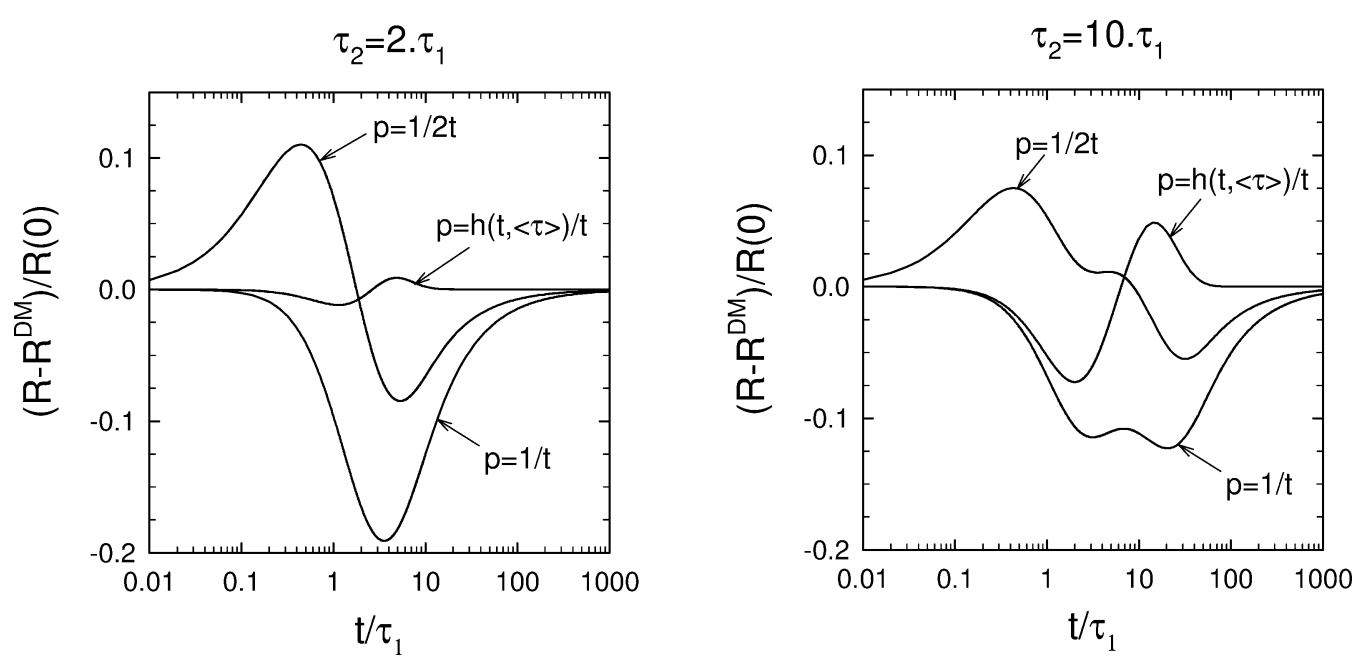

Fig. 4. Normalised difference between the exact relaxation function and the one predicted by the quasi-elastic approximation for two ratios $\tau_{2} / \tau_{1}$ in the different cases $p=0.5 / t, p=1 / t$, and $p=h(t) / t$. Two-phase material with "parallel" Maxwellian phases, $c_{2}=0.5$, and $k=1$.

are derived in Appendix A. In that case, taking $p=0.5 / t$ (i.e., $10^{w_{0}}=0.5$ ) in Eq. (12) gives better predictions than $p=1 / t$ at large $t / \tau_{1}$, but the slope at $t / \tau_{1}=0$ becomes simultaneously too steep. The direct method with a relation between $p$ and $t$ of the form $p=\alpha / t$, with $\alpha$ a constant scalar, seems therefore to be mainly limited to creep loading.

As for creep, a better way to treat relaxation loading with the quasi-elastic approximation is to choose a function $p=p(t)$ for which the direct method gives the exact response for a homogeneous material. Relation (12) is thus transformed into

$$
f(t) \simeq \hat{f}(p)_{p=h(t) / t},
$$

where the function $h(t)$ is adjusted with respect to the relaxation function of the single phase, Eq. (28):

$$
h(t)=\frac{t \mathrm{e}^{-t / \tau}}{\tau\left(1-\mathrm{e}^{-t / \tau}\right)} .
$$

It must be remarked that $h(t)$ now depends on the relaxation time $\tau$ of the considered phase. Thus, for the application to heterogeneous materials with phases exhibiting different $\tau_{i}, \tau$ in Eq. (32) could be replaced by an average quantity, say $\bar{\tau}$. Several definitions for $\bar{\tau}$ may be adopted. Among a few attempts, the best results were obtained here for $\bar{\tau}$ defined as $\bar{\tau}=\langle\tau\rangle$. Results are presented in Figs. 4 and 5. Clearly, the proposed treatment greatly improves the predictions at both local and global scales, as compared to the original proposition $p=10^{w_{0}} / t$. The relaxation functions quickly decrease in good agreement with the exact solutions, and the overall response is particularly close to the exact one. However, local response are not predicted as precisely as for creep. Indeed, as shown in Fig. 5, the relaxation of phase 1 is too slow whereas that of phase 2 is too fast. This may originate from the choice of a unique $\bar{\tau}$ for the two phases. Finally, it is worth noting that, as for creep, the proposed treatment also leads to the exact solution if $\tau_{1}=\tau_{2}$, whatever the value of $k$. The error introduced by the quasi-elastic approximation has clearly to be related to the local relaxation time ratio $\tau_{1} / \tau_{2}$.

\subsection{Other deformation paths}

The good agreement obtained for a relaxation loading shows that the direct inversion method is not limited to creep. For example, if the material is homogeneous and subjected to a constant strain rate loading of the form $\dot{\boldsymbol{\varepsilon}}(t)=\dot{\mathbf{E}}_{0} H(t)$, the stress response reads, for $t>0$

$$
\boldsymbol{\sigma}(t)=\frac{\dot{\mathbf{E}}_{0}}{\mathrm{~m}^{\mathrm{v}}}\left(1-\mathrm{e}^{-t / \tau}\right) \stackrel{\mathcal{L C}}{\longrightarrow} \hat{\boldsymbol{\sigma}}(p)=\frac{\dot{\mathbf{E}}_{0}}{\mathrm{~m}^{\mathrm{v}}} \frac{1}{1+\tau p} .
$$

Thus, the same function $h(t)$ as the one given in (32) can be used. As for relaxation, an average $\bar{\tau}$ must be defined for heterogeneous materials and here again, the exact solution is attained if all phases exhibit the same relaxation time. A similar procedure could also be used for more complex loading paths (cycling loading, for instance). 
(a)

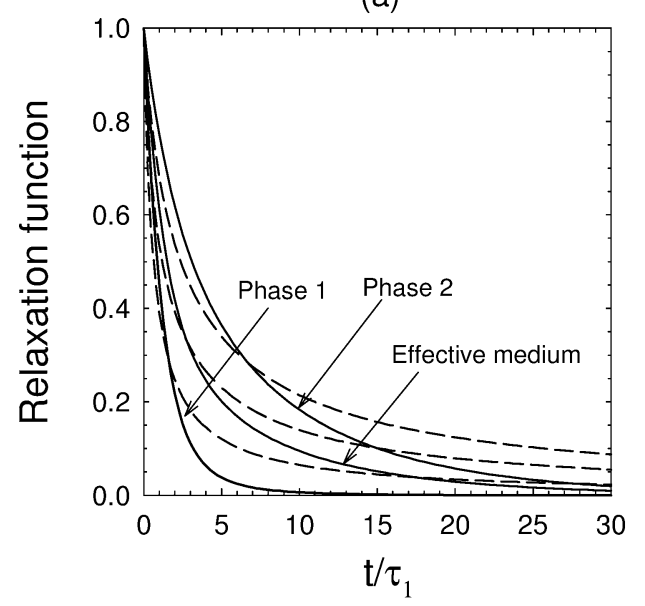

(b)

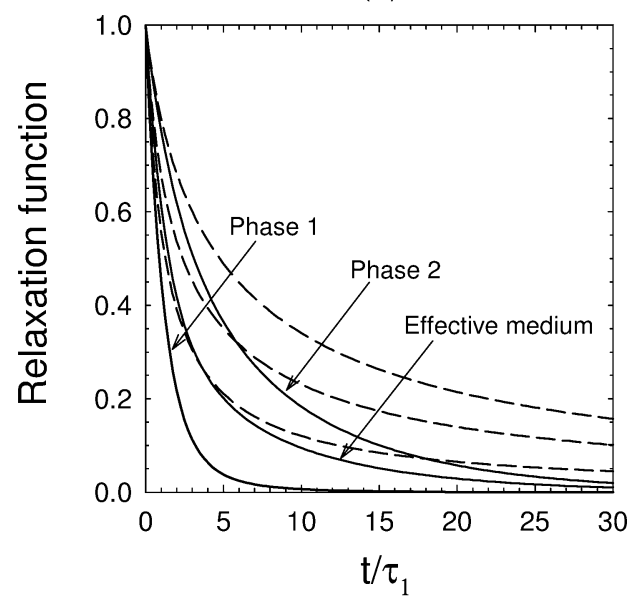

(c)

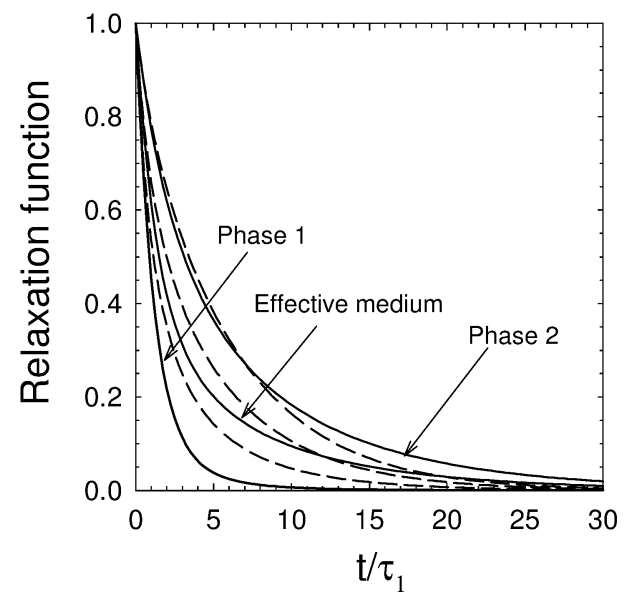

Fig. 5. Local $(r)$ and overall $(R)$ relaxation functions predicted by the quasi-elastic approximation (dashed lines) as compared to the exact results (continuous lines) for a two-phase material according to the self-consistent scheme and $\tau_{2} / \tau_{1}=10, c_{2}=0.5, k=1$, in the cases (a) $p=0.5 / t$, (b) $p=1 / t$, and (c) $p=h(t) / t$.

\subsection{Conclusion}

The application of the direct inversion method to simple isotropic materials with (linear) Maxwellian local behaviour has allowed us to analyse its domain of validity. First of all, it has been remarked that the initial Maxwellian character of the local behaviour is lost when the direct method is applied. In the case of creep loading, this results in an additional time-dependent local elastic compliance. Due to the shape of the single phase creep function, an improved treatment with $10^{w_{0}}=1$ has been proposed, which leads to the exact asymptotic regime for heterogeneous materials at $t=0$ and $t \rightarrow \infty$. Similarly, we have shown how other deformation paths can be treated by using a relation between $p$ and $t$ of the form $p=h(t) / t$, where the function $h(t)$ has to be adjusted on the response of a single phase material. In the case of relaxation, an average relaxation time $\bar{\tau}$ must however be defined and for this several choices are possible. It has been shown that the direct method provides the exact local and global responses if the relaxation times of the different phases are identical, and therefore its accuracy is expected to become worst with increasing spread of local relaxation times. A very good agreement at both local and global scales has been found in the presented numerical applications, suggesting that the direct method may be also very efficient for nonlinear problems. Since the inversion of the LC transform becomes immediate, the direct method is very powerful numerically. The counterpart of this is that, contrary to the collocation method, the accuracy of the method cannot be improved by means of 
improved numerical convergence. It has been noticed that the long range memory effects are still considered, that is the overall response is nowhere assumed to be Maxwellian, in accordance with Suquet (1985).

\section{The "quasi-elastic" approximation of the affine formulation}

\subsection{Consequences of the direct method}

We now go back to the nonlinear frame and study the application of the direct method in that context. The local constitutive equations have the form (1), i.e., they define a nonlinear Maxwell-type behaviour. According to the results obtained in Section 3, the overall creep responses are aproximated with $w_{0}=0$, whereas other choices are more appropriate for other loading paths. Whatever this choice, the symbolic homogenisation relations (9) now read

$$
\left\{\begin{array}{l}
\mathbf{Q}_{\zeta}(t)=\left\langle\mathbf{q}_{\zeta}(t): \mathbf{B}_{r}(t, \zeta)\right\rangle, \\
\mathbf{E}^{0}(t, \zeta)=\left\langle{ }^{t} \mathbf{B}_{r}(t, \zeta): \boldsymbol{\varepsilon}_{r}^{0}(t, \zeta)\right\rangle .
\end{array}\right.
$$

The unknown tensors $\mathbf{B}_{r}(t, \zeta)$ are obtained by solving the homogenisation problem in the LC space (first equation of (9)) and applying the variables change $p=h(t) / t$, with $h(t)=1$ for a creep loading. Equivalently, as shown above, homogenisation can be carried out directly in the time space. As for the prestrain field $\varepsilon_{r}^{0}(t, \zeta)$, it does not need this approximate inversion since it is explicitly defined in the time space according to (4) and (5). Nevertheless, it is emphasised that this field does not identify with the one that would be obtained with the "classical" affine formulation of Masson and Zaoui (1999) since the average stresses per phase are generally different. This observation is closely linked to the notion of linear comparison medium. Indeed, the new definition of the homogenised compliance and prestrain (34) corresponds to an effective behaviour that reads

$$
\mathbf{E}(t)=\mathbf{Q}_{\zeta}(t): \mathbf{\Sigma}(t)+\mathbf{E}^{0}(t, \zeta)
$$

It thus refers to a thermoelastic (with time-dependent characteristics), instead of thermoviscoelastic, linear comparison medium. That is why this approach is called "quasi-elastic", the convolution products of the initial problem being replaced by algebraic ones. Nevertheless, as explained above, it retains the essential features of the nonlinear viscoelastic behaviour. Particularly, the elastic and viscous behaviours are not treated separately during the transition scale, e.g., the linearised overall behaviour is not supposed to be of the Maxwell-type. Besides, the definition of the eigenstrains $\boldsymbol{\varepsilon}_{r}^{0}$ is left unchanged, which keeps its hereditary nature.

\subsection{The "quasi-elastic" affine procedure}

\subsubsection{Formulation}

Using the above results, the "quasi-elastic" affine procedure can be derived as follows. Let us consider, e.g., a macroscopic stress loading path $\boldsymbol{\Sigma}(t)$ on the time interval $[0, \zeta]$. We aim at the determination of the effective response $\mathbf{E}(\zeta)$. Due to the hereditary character of the behavior, we must assume that the problem was solved until $t=\zeta-\delta t$. The phase stress averages are thus known on $[0, \zeta-\delta t]$ and the unknowns are $\sigma_{r}(\zeta)$. For a given linearisation time $\zeta$, the linearised behavior reads, at any time $t \leqslant \zeta$,

$$
\left\{\begin{array}{l}
\boldsymbol{\varepsilon}_{r}(t)=\mathbf{q}_{\zeta}(t): \boldsymbol{\sigma}_{r}(t)+\boldsymbol{\varepsilon}_{r}^{0}(t, \zeta), \\
\mathbf{q}_{\zeta}(t)=\mathbf{s}+\mathbf{m}(\zeta) t \\
\boldsymbol{\varepsilon}_{r}^{0}(t, \zeta)=\int_{0}^{t} \dot{\boldsymbol{\varepsilon}}_{r}^{0}(\theta, \zeta) \mathrm{d} \theta, \\
\dot{\boldsymbol{\varepsilon}}_{r}^{0}(\theta, \zeta)=\mathbf{g}\left(\boldsymbol{\sigma}_{r}(\theta)\right)-\mathbf{m}(\zeta): \boldsymbol{\sigma}_{r}(\theta)
\end{array}\right.
$$

Due to the quasi-elastic approximation, it is only required to apply relation (36) for $t=\zeta$. The Hashin-Shtrikman-type homogenisation procedure leads to the following interaction equation

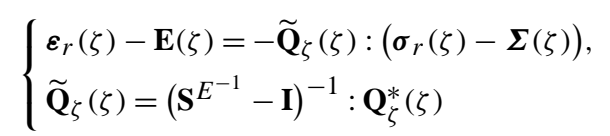

with $\widetilde{\mathbf{Q}}_{\zeta}$ the interaction tensor, $\mathbf{S}^{E}$ the Eshelby tensor and $\mathbf{Q}_{\zeta}^{*}$ the compliance of the reference medium which depends on the chosen homogenisation scheme. The quasi-elastic affine formulation is entirely defined by (34), (36) and (37). Thus, its 
implementation only requires the resolution of a set of nonlinear equations which reads $F\left(\sigma_{r}(\zeta)\right)=0$. Roughly, it reduces the CPU time by a factor 10 with respect to the resolution with use of the collocation method. The problem is solved with a classical fixed-point iterative algorithm. Note that an additional inner iterative resolution is required to solve (34) for the particular case of the self-consistent scheme.

\subsubsection{Comparison with former works}

A previous application of the quasi-elastic approximation (hereafter denoted TTW) was made by Turner et al. (1994) to derive the overall properties of nonlinear viscoelastic polycrystals. The differences between their formulation and our proposition are significant enough to be emphasised. Starting from the good agreement obtained with the quasi-elastic method in the linear case, for the particular case of a creep loading, as already mentioned by Laws and McLaughlin (1978), Yurner et al. (1994) proposed an empirical extension to the nonlinear case. Their proposed interaction equation, namely

$$
\dot{\boldsymbol{\varepsilon}}_{r}(t)-\dot{\mathbf{E}}(t)=-\widetilde{\mathbf{Q}}^{\mathrm{TG}}(t):\left(\dot{\boldsymbol{\sigma}}_{r}(t)-\dot{\boldsymbol{\Sigma}}(t)\right)-n \dot{\mathbf{Q}}^{\mathrm{TG}}(t):\left(\sigma_{r}(t)-\boldsymbol{\Sigma}(t)\right),
$$

where $\dot{\widetilde{\mathbf{Q}}}^{\mathrm{TG}}$ identifies in the viscoplastic regime with the accommodation tensor of the tangent model of Molinari et al. (1987), uses the assumption of a power-law viscous flow with a unique stress sensitivity coefficient $n$. It can be checked that such a law leads to a tangent description of the steady-state regime. This property is the sole justification of the proposition (38) which is, roughly speaking, an empirical interpolation between a purely elastic and a tangent viscoplastic regime. On the contrary, according to our approach, the local compliance in the steady-state limit is expressed as $\mathbf{q}(t)=\mathbf{s}+\mathbf{m} t$ with $\mathbf{m}$ independent on $t$ since $\lim _{t \rightarrow+\infty} \dot{\boldsymbol{\sigma}}_{r}(t)=0$. The behaviour becomes purely viscoplastic (or nonlinear viscous) and the formulation obtained, still referring to a thermoelastic linear medium, identifies with the affine procedure for viscoplastic material which differs significantly from the tangent model (Masson et al., 2000). Especially, for highly nonlinear behaviours, the tangent model tends to the Reuss bound (Lebensohn and Tomé, 1993), which is not the case of the affine procedure. However, according to Molinari (2002), the tangent model may be seen as one particular procedure within a more general approach.

Apart from this remark which only concerns the purely nonlinear viscous regime, a specific difference exists in the transient regime where the viscoelastic coupling takes place. Indeed, the TTW approach does not take into account the influence of the whole stress history, since the behaviour is linearised as follows

$$
\boldsymbol{\varepsilon}_{r}(\zeta)=\left(\mathbf{s}+\mathbf{m}^{\mathrm{sec}}(\zeta) \zeta\right): \boldsymbol{\sigma}_{r}(\zeta)
$$

with $\mathbf{m}^{\text {sec }}$ the viscous linearised secant compliance. For a power-law viscous flow with stress sensitivity $n$, Eq. (39) gives (Hutchinson, 1976)

$$
\left\{\begin{array}{l}
\boldsymbol{\varepsilon}_{r}(\zeta)=(\mathbf{s}+\mathbf{m}(\zeta) \zeta): \boldsymbol{\sigma}_{r}(\zeta)+\boldsymbol{\varepsilon}_{r}^{0}(\zeta), \\
\boldsymbol{\varepsilon}_{r}^{0}(\zeta)=(1-n) \mathbf{m}^{\mathrm{sec}}(\zeta) \zeta: \boldsymbol{\sigma}_{r}(\zeta)
\end{array}\right.
$$

with the tangent compliance $\mathbf{m}=n \mathbf{m}^{\mathrm{sec}}$. It is to be stressed that the TTW formulation (40) differs from the affine one presented in this paper, since it relies on an instantaneous definition of the stress-free strain $\boldsymbol{\varepsilon}_{r}^{0}(\zeta)$ whereas the quasi-elastic affine approach accounts for the hereditary nature of the behaviour. Consequently, the TTW approach neglects the cumulated influence of the whole stress history on the current linearised behaviour.

\section{Application to polycrystals}

We now consider the elastoviscoplastic behaviour of various polycrystals. We assume that the material is made of randomly distributed grains with an equiaxed shape. Consequently, all the grains with the same crystallographic orientation define a phase with a given volumic fraction and an isotropic distribution. In the following, we compare the elastoviscoplastic response of this material as predicted by the self-consistent "classical" affine formulation (Masson and Zaoui, 1999) and the self-consistent quasi-elastic affine treatment proposed here.

\subsection{Constitutive behaviour}

We assume that the viscoplastic deformation is only due to dislocation glide on crystallographic slip systems. According to the kinematics of plasticity, it follows:

$$
\mathbf{g}=\sum_{s} \dot{\gamma}_{s} \mathbf{R}_{s}
$$


The Schmid tensor $\mathbf{R}_{s}$, characterizing the orientation of the slip system $(s)$, is given by

$$
\mathbf{R}_{s}=\frac{1}{2}\left(\mathbf{n}_{s} \otimes \mathbf{m}_{s}+\mathbf{m}_{s} \otimes \mathbf{n}_{s}\right)
$$

with $\mathbf{n}_{s}$ and $\mathbf{m}_{s}$ the unit vectors normal to the slip plane and along the slip direction. In the sequel, we consider a classical power-law type behaviour of the form

$$
\dot{\gamma}_{s}=\dot{\gamma}_{s}^{0}\left(\frac{\tau_{s}}{\tau_{s}^{0}}\right)^{n},
$$

with $\dot{\gamma}_{s}^{0}$ the reference slip rate and $\tau_{s}^{0}$ the reference shear stress of system $(s)$ and $n$ the material stress sensitivity. The resolved shear stress is defined as $\tau_{s}=\mathbf{R}_{s}: \sigma$. For simplicity, the reference shear stresses and Schmid tensors will be kept constant here. In other words, we shall not consider hardening effects and texture evolution.

We consider polycrystals with two types of crystalline structures: face-centered cubic (FCC) and hexagonal close-packed (HCP). For FCC polycrystals, glide occurs on the twelve octahedral slip systems $\{111\}\langle 110\rangle$ with the same reference slip rate $\dot{\gamma}_{s}^{0}$ and shear stresses $\tau_{s}^{0}$. Such polycrystals consequently obey a weakly anisotropic plastic behaviour. For HCP polycrystals, we assume three different slip modes to be active: prismatic $\{1 \overline{1} 00\}\langle 11 \overline{2} 0\rangle$, basal $\{0001\}\langle 11 \overline{2} 0\rangle$ and first-order pyramidal $\langle\mathbf{c}+\mathbf{a}\rangle\{1 \overline{1} 01\}\langle 1 \overline{2} 1 \overline{3}\rangle$. All systems present the same reference slip rate but we consider a ratio of 6 on the reference shear stresses between the prismatic system and the other ones. The prismatic system is taken as the easy-glide system. Contrarily to the FCC case, these polycrystals present a highly anisotropic plastic behaviour. They can be thought as zirconium-type polycrystals. For both kinds of polycrystals, we assume a uniform power-law exponent $n=5$, which is representative of hightemperature deformation mechanisms. Besides, the elasticity is isotropic with a Poisson ratio of $1 / 3$. For the FCC case, we have $\tau^{0} / E_{y}=10^{-3}$ and for the HCP case, we have $2 \tau_{\mathrm{pr}}^{0} / E_{y}=10^{-3}$ (where the index pr stands for "prismatic") with $E_{y}$ the Young modulus. Finally, the polycrystals under consideration here are untextured.

\subsection{Creep responses}

We performed computations for tensile creep tests with instantaneous loading at $t=0$. Fig. 6 shows the evolution of the overall equivalent creep strain $E_{\mathrm{eq}}$, normalised by the equivalent elastic strain $E_{\mathrm{eq}}^{\mathrm{e}}$, for FCC and HCP polycrystals. It can be observed that the affine effective response predicted with the use of the direct method with $p=1 / t$ lies very close to the one obtained with the collocation method in the transient and steady-state regimes. Thus, our approach is shown to be relevant even in the case of a highly anisotropic viscoplastic behaviour (i.e., with a high contrast on the relaxation times). To help understanding the accuracy of the "quasi-elastic" approximation, the results of a decoupled model identifying separately the elastic strains and the viscous strains are also reported in Fig. 6. This approach assumes that the overall behaviour is of the Maxwell-type. Consequently, in the case of a creep test with an instantaneous elastic loading at $t=0$, it is unable to describe the transient regime and leads directly for $t>0$ to the steady-state viscous response. Comparing the FCC and HCP cases, it

(a)

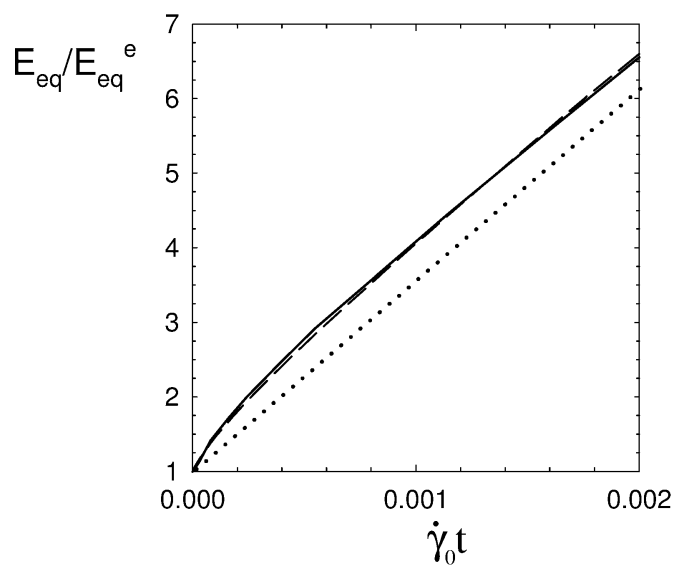

(b)

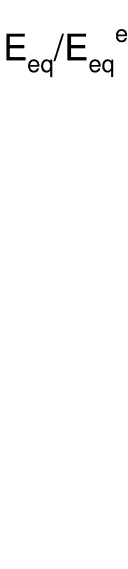

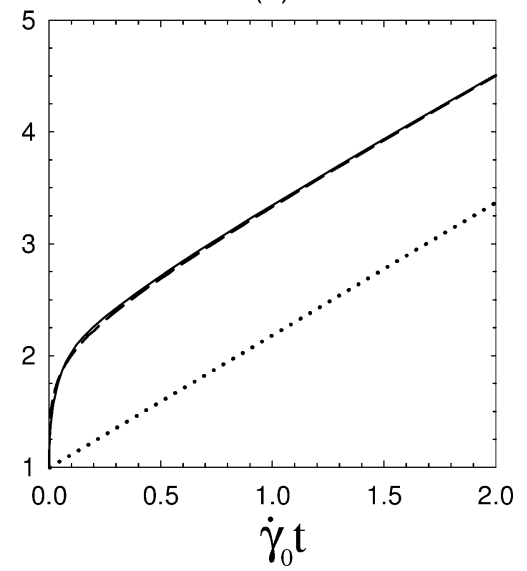

Fig. 6. Normalised overall equivalent strain for a tensile creep test for (a) $\operatorname{FCC}\left(\Sigma_{33}=4 \tau^{0}\right)$ and (b) $\mathrm{HCP}\left(\Sigma_{33}=2 \tau_{\mathrm{pr}}^{0}\right)$ polycrystals as predicted by the affine model (continuous line), its quasi-elastic approximation (dashed lines) with $p=1 / t$ and a decoupled (Maxwell-type) model (dotted lines). 

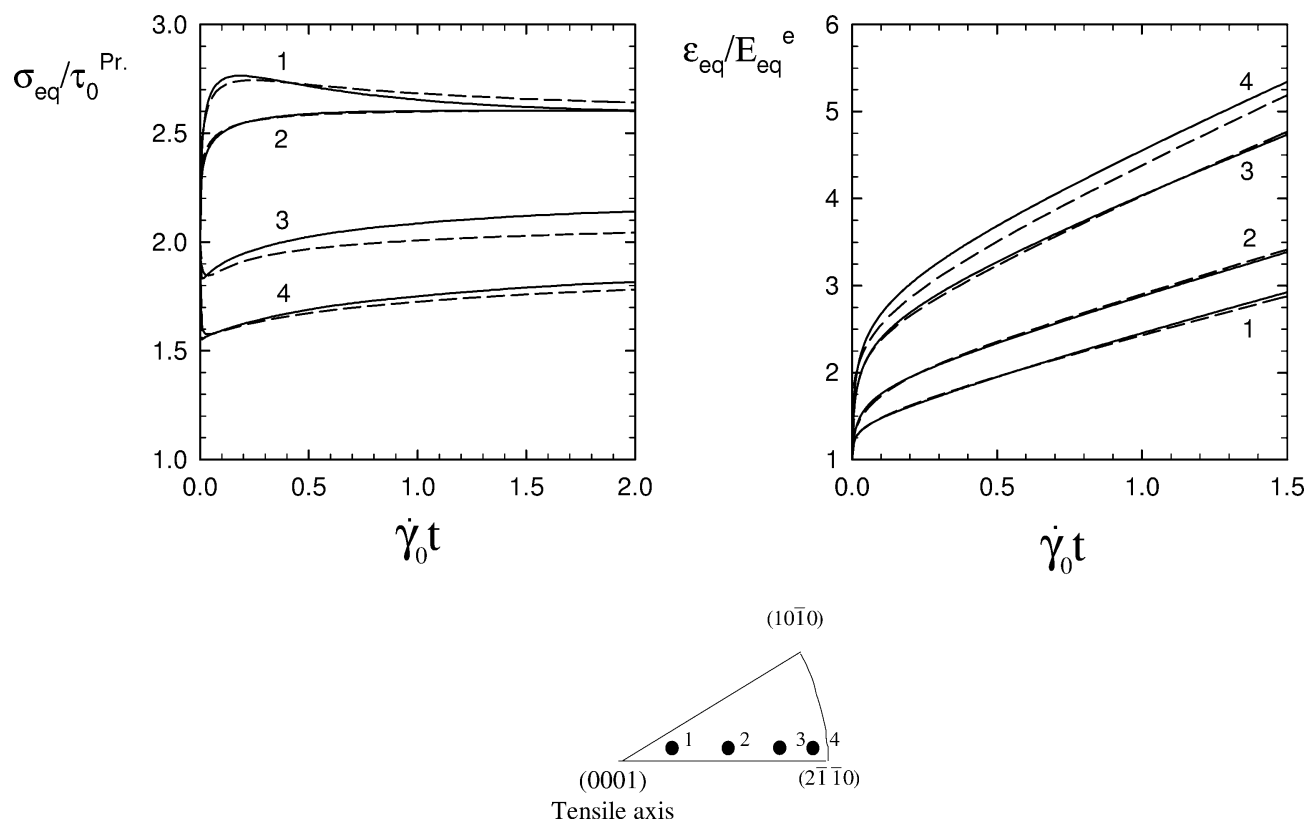

Fig. 7. Intraphase average stress and strain responses for four different orientations during a tensile creep test $\left(\Sigma_{33}=2 \tau_{\text {pr }}^{0}\right)$ for an HCP polycrystal as predicted by the affine model (continuous line) and its quasi-elastic approximation (dashed lines) with $p=1 / t$.

is observed that the discrepancy between the "full" affine scheme and this crude approximation increases with the viscoplastic anisotropy whereas the "quasi-elastic" formulation remains valid in both cases.

For the HCP polycrystal, it is particularly interesting to study the predictions of the phase field averages, since the interphase fields heterogeneity is very pronounced. Due to the transverse isotropy of the phase responses around the loading direction, computations can be restricted to one spherical standard triangle. The stress and strain responses are reported in Fig. 7 for four orientations in the standard triangle. The evolution of the stresses shows that the transient response of the "soft" and "hard" orientations is correctly captured by our approach and that the relaxation times are very similar in the two approaches. It can be stressed that the decoupled model would predict, as for the effective behaviour, a constant strain rate (versus time) in each phase for $t>0$ and therefore also a constant stress. Apart from this remark, it is seen that the large interphase strain heterogeneity, which is physically relevant with respect to the grain-to-grain incompatibility, is satisfactorily restituted with the present proposition. It is recalled that, in the viscoplastic regime, this formulation coincides with the affine formulation. From that point of view, our approximate treatment is expected to be more accurate than former approximate approaches which are known to lead in the steady-state regime either to the Taylor bound (Weng, 1981, 1993), as shown by Masson and Zaoui (1999), or the Reuss bound (Turner et al., 1994) for a highly nonlinear viscoplastic potential. The good agreement of our treatment with the affine formulation has important practical implications. Indeed, since the computation time is divided by a factor of about 10, it allows an identification of the model on a wide experimental database and makes it a powerful tool for the study of polycrystal plasticity (see Brenner, 2001; Letouzé et al., 2002; Brenner et al., 2002).

\subsection{Relaxation responses}

As shown previously, the quasi-elastic approach has not to be limited to creep. As a first illustration, the response of the FCC polycrystal to a uniaxial relaxation loading has been studied. According to the results obtained in Section 3, we apply the direct method with $p=h(t) / t$ and $h(t)$ defined by (32). For that goal, it is necessary to determine a reference relaxation time for the polycrystal. This step is not straightforward due to the local anisotropy of the material. Among other possibilities, we defined at each linearisation time $\zeta$ the "relaxation time" of each phase $\tau_{r}$ and the reference relaxation time $\bar{\tau}$ by

$$
\tau_{r}=\frac{s_{i j i j}^{r}}{m_{i j i j}^{r}}, \quad \bar{\tau}=\left\langle\tau_{r}\right\rangle .
$$

Fig. 8 shows the overall stress responses according to the direct method with $p=h(t) / t$ and $p=t$ and to the classical affine formulation. It can be seen, as expected, that the choice of $p=h(t) / t$ leads to a better agreement in the transient regime, especially at short reduced times, than the choice $p=1 / t$. Though our definition of the reference relaxation time is very simple 


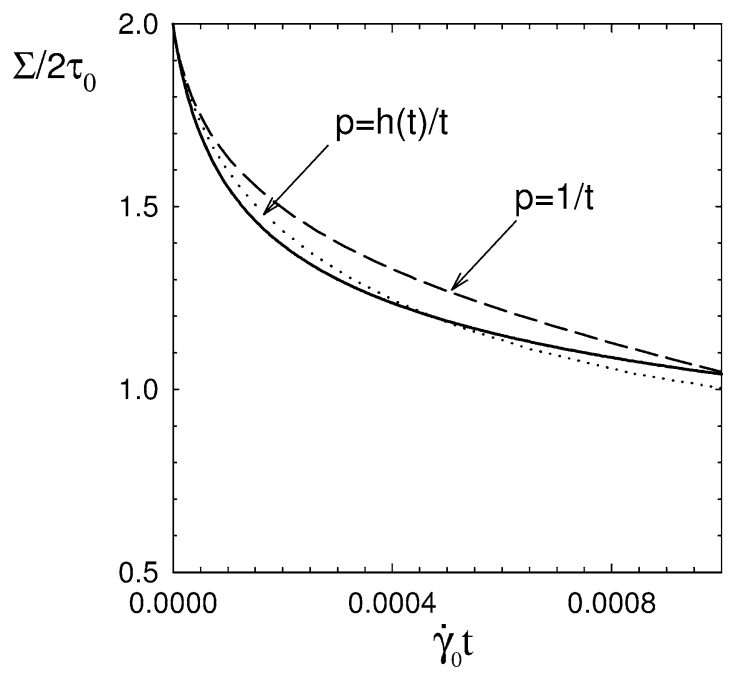

Fig. 8. Normalised overall equivalent stress during a tensile relaxation test $\left(E_{33}=4 \tau^{0} / E_{y}\right)$ for a FCC polycrystal as predicted by the affine model (continuous line) and its quasi-elastic approximation (dashed lines) with $p=1 / t$ et $p=h(t) / t$.

and certainly not optimal, this result shows a simple but efficient way to manage with another type of loading than creep with an appropriate definition of the function $h(t)$. Extension to constant strain rate behaviour, for instance, is straightforward (see Section 3.4). To the best of our knowledge, the proposed approach is the first to extend the quasi-elastic treatment to other loadings than creep.

In view of these results, it can be appreciated that our approach reconciles an affine formulation which is known to be one of the most accurate at the time for rate-dependent plasticity (Masson and Zaoui, 1999) with an efficient numerical resolution which renders the computation as simple as for any other nonlinear homogenisation scheme since it uses a thermoelastic linear comparison medium. Additionally, it is recalled that this formulation does not imply any restriction on the chosen linear homogenisation scheme (and so on the form of the interaction equation) or on the form of the nonlinear Maxwell-type behaviour of the constituents: it can be used with more refined forms of the local viscoplastic potential than the one used in this section.

\section{Conclusion}

Several major difficulties have to be overcome for the application of homogenisation techniques to nonlinear viscoelastic heterogeneous materials. First of all, a linearisation procedure must be defined. In the present study, the proposition of Rougier et al. (1994) has been adopted. Next, per phase uniform linear viscoelastic compliances have to be calculated, and for this a reference stress must be defined. The classical (and most simple) choice defining the linearised compliances with respect to the stress phase average has been chosen here. Finally, the mechanical fields obtained in the Laplace-Carson space at each linearisation time have to be transformed back into the physical time space. This last point is more "technical", but it has a great practical importance because it renders the homogeneisation scheme quite CPU time-consuming.

Although an accurate inversion can be carried out numerically, the application to anisotropic polycrystals, with a necessarily associated step of coefficients identification, is hardly workable. For this, we have reconsidered the approximate direct inversion method initially proposed by Schapery (1962) which leads to a reduction of CPU time by one order of magnitude. Its accuracy has been first investigated on a linear heterogeneous material with maxwellian phases. It has been shown that the method is exact if the phases exhibit identical relaxation times, and the improved treatment proposed here renders the direct method applicable to any loading path. Applied to nonlinear polycrystals in the framework of the "classical affine" linearisation procedure, the direct method is shown to describe the exact asymptotic behaviour at $t=0$ and $t \rightarrow \infty$. The initial thermoviscoelastic linear comparison material is transformed into a thermoelastic one, so that homogenisation can be completely carried out in the physical time space. Moreover, the long range memory effects associated to the viscoelastic coupling are still taken into account. Illustrative examples for FCC and HCP nonlinear polycrystals show that the direct method predicts almost the same mechanical responses in creep and relaxation as the collocation treatment at both local and global scales, and therefore reconciles the accuracy of the affine formulation with a very efficient numerical resolution.

It is emphasized that the "direct method" can also be used in the context of the generalised affine linearisation proposed by Pouya and Zaoui (1999) and/or when the reference stress accounts for intraphase heterogeneity. These aspects are currently under development. 


\section{Acknowledgement}

One of us (R.B.) gratefully acknowledges the Commissariat à l'Energie Atomique for the partial funding of this work.

\section{Appendix. Analytical derivation of the creep and relaxation behaviour of the two-phase material of Section 3}

The derivation of the original time functions of the Laplace-Carson tranformed functions $\widehat{F}(p), \hat{f}_{i}(p), \widehat{R}(p)$ and $\hat{r}_{i}(p)$ defined in (25) and (30), where $\hat{\mu}_{i}(p)$ and $\hat{\tilde{\mu}}(p)$ are given by (16) and (22) respectively (with the convention $\left.\tau_{1}<\tau_{2}\right)$, can make use of the Mellin-Bromwich inversion formula

$$
f(t)=\frac{1}{2 \mathrm{i} \pi} \int_{\Delta} \frac{\hat{f}(p)}{p} \mathrm{e}^{p t} \mathrm{~d} p,
$$

where the straight line $\Delta$, parallel to the y-axis, must leave all the poles and critical points of $\hat{f}(p) / p$ at left. This line $\Delta$ may be complemented on its left by a half-circle centered on it at $y=0$ with a radius growing to infinity; the contribution of this half-circle to the integral in (A.1) vanishes according to Jordan's lemma. Due to the term $[P(p)]^{1 / 2}$ in $(22)$, which needs to be uniformly defined, a cut must be defined on the real negative axis between $-1 / \theta_{1}$ and $-1 / \theta_{2}$, with $-1 / \tau_{1}<-1 / \theta_{1}<-1 / \theta_{2}<$ $-1 / \tau_{2}<0$. The computation then reduces to that of some residues on the $\mathrm{x}$-axis outside the cut $\left[-1 / \theta_{1},-1 / \theta_{2}\right]$ and of an integral on this interval.

The searched functions read as follows (when $k \neq 1$ and $k \neq \tau_{1} / \tau_{2}$ ):

- Creep

$$
\begin{aligned}
& f_{1}(t)=\frac{\mathrm{m}_{1}^{\mathrm{e}} \tau_{1} \tau_{2}}{4(1-c)\left(k \tau_{2}-\tau_{1}\right)}\left\{\frac{k(2-5 c)}{\tau_{1}}+\frac{5 c-7}{\tau_{2}}-\frac{5 k\left(\tau_{2}-\tau_{1}\right)}{\left(k \tau_{2}-\tau_{1}\right) \tau_{1}}+\frac{K}{\tau_{1}\left(\theta_{1} \theta_{2}\right)^{1 / 2}}\left[\frac{\theta_{1}+\theta_{2}}{2}+\frac{\tau_{1}\left(\tau_{2}-\tau_{1}\right)}{k \tau_{2}-\tau_{1}}\right]\right. \\
& \left.+\left[\frac{k(2-5 c)}{\tau_{1}}+\frac{5 c-7}{\tau_{2}}+\frac{K}{\left(\theta_{1} \theta_{2}\right)^{1 / 2}}\right] \frac{t}{\tau_{1}}-\frac{K}{\pi}\left[\frac{J_{1}}{\tau_{1}}+\frac{\left(\tau_{2}-\tau_{1}\right)\left(J_{2}-J_{3}\right)}{k \tau_{2}-\tau_{1}}\right]\right\}, \\
& b=\frac{k \tau_{2}-\tau_{1}}{(k-1) \tau_{1} \tau_{2}}, \quad J_{1}=\int_{-1 / \theta_{1}}^{-1 / \theta_{2}} \frac{[-P(x)]^{1 / 2}}{x^{2}} \mathrm{e}^{t x} \mathrm{~d} x, \\
& J_{2}=\int_{-1 / \theta_{1}}^{-1 / \theta_{2}} \frac{[-P(x)]^{1 / 2}}{x} \mathrm{e}^{t x} \mathrm{~d} x, \quad J_{3}=\int_{-1 / \theta_{1}}^{-1 / \theta_{2}} \frac{[-P(x)]^{1 / 2}}{x+b} \mathrm{e}^{t x} \mathrm{~d} x, \\
& f_{2}(t)=\frac{\mathrm{m}_{1}^{\mathrm{e}} \tau_{1} \tau_{2}}{4 c k\left(\tau_{1}-k \tau_{2}\right)}\left\{-\frac{k(2+5 c)}{\tau_{1}}+\frac{5 c-3}{\tau_{2}}-\frac{5 k\left(\tau_{2}-\tau_{1}\right)}{\left(k \tau_{2}-\tau_{1}\right) \tau_{2}}+\frac{K}{\tau_{2}\left(\theta_{1} \theta_{2}\right)^{1 / 2}}\left[\frac{\theta_{1}+\theta_{2}}{2}+\frac{k \tau_{2}\left(\tau_{2}-\tau_{1}\right)}{k \tau_{2}-\tau_{1}}\right]\right. \\
& \left.+\left[-\frac{k(2+5 c)}{\tau_{1}}+\frac{5 c-3}{\tau_{2}}+\frac{K}{\left(\theta_{1} \theta_{2}\right)^{1 / 2}}\right] \frac{t}{\tau_{2}}-\frac{K}{\pi}\left[\frac{J_{1}}{\tau_{2}}+\frac{k\left(\tau_{2}-\tau_{1}\right)\left(J_{2}-J_{3}\right)}{k \tau_{2}-\tau_{1}}\right]\right\}, \\
& F(t)=(1-c) f_{1}(t)+c f_{2}(t)=\frac{\mathrm{m}_{1}^{\mathrm{e}}}{4 k}\{k(2-5 c)+5 c-3 \\
& \left.+\left[\frac{k(2-5 c)}{\tau_{1}}+\frac{5 c-3}{\tau_{2}}\right] t+\frac{K}{\left(\theta_{1} \theta_{2}\right)^{1 / 2}}\left(\frac{\theta_{1}+\theta_{2}}{2}+t\right)-\frac{K J_{1}}{\pi}\right\} .
\end{aligned}
$$

- Relaxation

$$
\begin{aligned}
& r_{1}(t)=\frac{1}{3 \mathrm{~m}_{1}^{\mathrm{e}}(1-c)}\left\{\langle 3-5 c\rangle \mathrm{e}^{-t / \tau_{1}}+\frac{K\left(J_{4}-J_{3}\right) \tau_{1} \tau_{2}}{2 \pi\left(\tau_{2}-\tau_{1}\right)}\right\}, \\
& \langle x\rangle=\frac{|x|+x}{2}, \quad J_{4}=\int_{-1 / \theta_{1}}^{-1 / \theta_{2}} \frac{[-P(x)]^{1 / 2}}{x+1 / \tau_{1}} \mathrm{e}^{t x} \mathrm{~d} x, \\
& r_{2}(t)=\frac{1}{3 \mathrm{~m}_{1}^{\mathrm{e}} c}\left\{\langle 5 c-2\rangle k \mathrm{e}^{-t / \tau_{2}}-\frac{K\left(J_{5}-J_{3}\right) \tau_{1} \tau_{2}}{2 \pi\left(\tau_{2}-\tau_{1}\right)}\right\}, \quad J_{5}=\int_{-1 / \theta_{1}}^{-1 / \theta_{2}} \frac{[-P(x)]^{1 / 2}}{x+1 / \tau_{2}} \mathrm{e}^{t x} \mathrm{~d} x,
\end{aligned}
$$




$$
R(t)=(1-c) r_{1}(t)+c r_{2}(t)=\frac{1}{3 \mathrm{~m}_{1}^{\mathrm{e}}}\left\{\langle 3-5 c\rangle \mathrm{e}^{-t / \tau_{1}}+\langle 5 c-2\rangle k \mathrm{e}^{-t / \tau_{2}}+\frac{K\left(J_{4}-J_{5}\right) \tau_{1} \tau_{2}}{2 \pi\left(\tau_{2}-\tau_{1}\right)}\right\} .
$$

Note that the integrals $J_{1}$ to $J_{5}$ may be expressed as functions of $\Phi_{1}$, degenerate hypergeometric series in two variables (Gradshteyn and Ryzhik, 1965). They have been evaluated numerically for the illustrations reported in Section 3.

When $k=1$, Eqs. (A.2) to (A.7) can still be used with $K=5$ and $J_{3}=0$.

\section{References}

Beurthey, S., Zaoui, A., 2000. Structural morphology and relaxation spectra of viscoelastic heterogeneous materials. Eur. J. Mech. A Solids 19, $1-16$.

Brenner, R., 2001. Influence de la microstructure sur le comportement en fluage thermique d'alliages de zirconium : analyse experimentale et mise en oeuvre de méthodes d'homogénéisation. Ph.D. thesis, Université Paris XIII, France.

Brenner, R., Béchade, J.-L., Castelnau, O., Bacroix, B., 2002. Thermal creep of Zr-Nb1\%-O alloys: experimental analysis and micromechanical modelling. J. Nucl. Mater., to appear.

Brenner, R., Castelnau, O., Gilormini, P., 2001. A modified affine theory for the overall properties of nonlinear composites. C. R. Acad. Sci. Paris Sér. Ilb 329, 649-654.

Brown, G.M., 1970. A self-consistent polycrystalline model for creep under combined stress state. J. Mech. Phys. Solids 18, 367-381.

Eshelby, J.D., 1957. The determination of the elastic field of an ellipsoidal inclusion, and related problems. Proc. Roy. Soc. London Ser. A 241, 376-396.

Gradshteyn, I.S., Ryzhik, I.M., 1965. Table of Integrals, Series and Products. Academic Press, New York.

Harren, S.V., 1991. The finite deformation of rate-dependent polycrystals-II. A comparison of the self-consistent and Taylor methods. J. Mech. Phys. Solids 39, 361-383.

Hill, R., 1965. Continuum micro-mechanics of elastoplastic polycrystals. J. Mech. Phys. Solids 13, 89-101.

Hutchinson, J.W., 1976. Bounds and self-consistent estimates for creep of polycrystalline materials. Proc. Roy. Soc. London Ser. A 348, 101127.

Kröner, E., 1961. Zur plastischen Verformung des Vielkristalls. Acta Metall. 9, 155-161.

Laws, N., McLaughlin, R., 1978. Self-consistent estimates for the viscoelastic creep compliances of composite materials. Proc. Roy. Soc. London Ser. A 359, 251-273.

Lebensohn, R.A., Tomé, C.N., 1993. A self-consistent anisotropic approach for the simulation of plastic deformation and texture development of polycrystals: application to zirconium alloys. Acta Metall. 41, 2611-2624.

Letouzé, N., Brenner, R., Castelnau, O., Béchade, J.-L., Mathon M.-H., 2002. Residual strain distribution in Zircaloy-4 measured by neutron diffraction and estimated by homogenization techniques. Scripta Mater., to appear.

Mandel, J., 1966. Mécanique des Milieux Continus. Gauthier-Villars, Paris.

Masson, R., Bornert, M., Suquet, P., Zaoui, A., 2000. An affine formulation for the prediction of the effective properties of nonlinear composites and polycrystals. J. Mech. Phys. Solids 48, 1203-1227.

Masson, R., Zaoui, A., 1999. Self-consistent estimates for the rate-dependent elastoplastic behavior of polycrystalline materials. J. Mech. Phys. Solids 47, 1543-1568.

Molinari, A., 2002. Averaging models for heterogeneous viscoplastic and elastic viscoplastic materials. J. Eng. Mat. Tech. 124, 62-70.

Molinari, A., Ahzi, S., Kouddane, R., 1997. On the self-consistent modeling of elastic-plastic behavior of polycrystals. Mech. Materials 26, 43-62.

Molinari, A., Canova, G.R., Ahzi, S., 1987. A self-consistent approach of the large deformation polycrystal viscoplasticity. Acta Metall. 35, 2983-2994.

Nemat-Nasser, S., Obata, M., 1986. Rate-dependent finite elstoplastic deformation of polycrystals. Proc. R. Soc. London Ser. A 407, $343-375$.

Paquin, A., Sabar, H., Berveiller, M., 1999. Integral formulation and self-consistent modelling of elastoviscoplastic behavior of heterogeneous materials. Arch. Appl. Mech. 69, 14-35.

Ponte Castañeda, P., 1991. The effective mechanical properties of nonlinear isotropic composites. J. Mech. Phys. Solids 39, 45-71.

Ponte Castañeda, P., 1996. Exact second-order estimates for the effective mechanical properties of nonlinear composite materials. J. Mech. Phys. Solids 44, 827-862.

Pouya, A., Zaoui, A., 1999. Linéarisation et homogénéisation en viscoélasticité. C. R. Acad. Sci. Paris Sér. Ilb 327, $365-370$.

Rougier, Y., Stolz, C., Zaoui, A., 1993. Représentation spectrale en viscoélasticité linéaire des matériaux hétérogènes. C. R. Acad. Sci. Paris Sér. II 316, 1517-1522.

Rougier, Y., Stolz, C., Zaoui, A., 1994. Self-consistent modelling of elastic-viscoplastic polycrystals. C. R. Acad. Sci. Paris Sér. II 318, $145-151$.

Schapery, R.A., 1962. Approximate methods of transform inversion for viscoelastic stress analysis. Proc. U.S. Nat. Congr. Appl. Mech. ASME 4th 2, 1075-1085.

Suquet, P., 1985. Elements of homogenization for inelastic solid mechanics. In: Sanchez-Palencia, E., Zaoui, A. (Eds.), Homogenization Techniques for Composite Media. Springer-Verlag, Berlin, pp. 193-278.

Suquet, P., 1993. Overall potentials and extremal surfaces of power law or ideally plastic composites. J. Mech. Phys. Solids 41, 981-1002.

Turner, P.A., Tomé, C.N., 1993. Self-consistent modeling of visco-elastic polycrystals: application to irradiation creep and growth. J. Mech. Phys. Solids 41, 1191-1211. 
Turner, P.A., Tomé, C.N., Woo, C.H., 1994. Self-consistent modelling of nonlinear visco-elastic polycrystals: an approximate scheme. Phil. Mag. A 70, 689-711.

Weng, G.J., 1981. Self-consistent determination of time dependent behavior of metals. J. Appl. Mech. 48, 41-46.

Weng, G.J., 1993. A self-consistent relation for time-dependent creep of polycrystals. Int. J. Plasticity 9, 181-198.

Zaoui, A., 1972. Effets de la désorientation des grains sur la viscoplasticité de polycristaux C.F.C. Int. J. Solids Structures 8, 1089-1101.

Zaoui, A., Raphanel, J.L., 1991. Investigation of inter- and intragranular plastic inhomogeneity. In: Lowe, T.C., Rollett, A.D., Follansbee, P.S., Daehn, G.S. (Eds.), Modeling the Deformation of Crystalline Solids. TMS, pp. 51-59. 